\title{
Gelation Mechanism of Erythromycin Ethylsuccinate During Crystallization
}

\author{
Xiandong Su $\mathrm{Su}^{1,2} \cdot$ Zhenguo Gao $^{1,2,3} \cdot$ Ying Bao $^{1,2} \cdot$ Miao Guan $^{1} \cdot$ Sohrab Rohani ${ }^{3} \cdot$ Qiuxiang Yin $^{1,2} \cdot$ Hongxun Hao $^{1,2}$. \\ Chuang Xie ${ }^{1,2}$. Jingkang Wang ${ }^{1,2}$
}

Received: 28 February 2018 / Revised: 11 April 2018 / Accepted: 26 April 2018 / Published online: 28 May 2018

(c) The Author(s) 2018

\begin{abstract}
In this paper, the gelation mechanism of erythromycin ethylsuccinate (EES) during crystallization is investigated for the first time. The generated semisolid gel-like phase exhibited a 3D fibrillar network morphology and the typical rheological properties of gels. The fibers inside the gel-like phase were confirmed to be new types of EES solvates using powder X-ray diffraction, thermogravimetric analysis/differential scanning calorimetry, and gas chromatography. The gelation and crystallization regions in EES-1-propanol solid-liquid phase diagram were determined. Analyses of solvent parameters showed that moderate solvent polarity may promote EES gelation. Fourier transform infrared spectra, nuclear magnetic resonance spectra, and scanning electron microscopy analyses indicated that through intermolecular hydrogen bonds, EES and solvent molecules assembled into fibers via crystallographic mismatch branching growth. The fibers intertwined into a 3D network microstructure and formed a gel-like phase, completely immobilizing the solution.
\end{abstract}

Keywords Gelation $\cdot$ Solvate $\cdot$ Crystallization $\cdot$ Phase diagram $\cdot$ Molecular self-assembly

\section{Introduction}

Solution crystallization is widely used in the production of pharmaceutical crystals. During the crystallization of active pharmaceutical ingredients (APIs) under various conditions, several studies have reported the occurrence of gelation phenomenon [1, 2]. Gelation of some APIs, such as clopidogrel sulfate [3] and valsartan [4], is rather likely to form a gel-like phase in their supersaturated state, which is an unwanted process in industrial crystallization. However, gelation may have some advantages. New perspectives

Electronic supplementary material The online version of this article (https://doi.org/10.1007/s12209-018-0163-5) contains supplementary material, which is available to authorized users.

Ying Bao

yingbao@tju.edu.cn

1 School of Chemical Engineering and Technology, Tianjin University, Tianjin 300072, China

2 Collaborative Innovation Centre of Chemical Science and Engineering (Tianjin), Tianjin 300072, China

3 Department of Chemical and Biochemical Engineering, Western University, London, ON N6A 5B9, Canada propose that gelation allows APIs to be used as molecular gel dosage forms and allows the control of crystal habits of APIs. Hence, the study of APIs gelation and the gelation mechanism is of great importance.

Gelation refers to a phenomenon where, under certain conditions, the solute molecules in a solution self-assemble into a 3D network structure that immobilizes the whole liquid phase [5]. At the macroscopic scale, the gelled solution loses its flowability and turns into a semisolid soft matter. Gelation is driven by the assembly of solute molecules through multiple reversible noncovalent interactions [6-8], including hydrogen bonds, $\pi-\pi$ stacking, van der Waals forces, electrostatic, dipole-dipole, and hydrophobic interactions.

Similar to crystallization, gelation is also a common phase separation phenomenon. During crystallization, macroscopic phase separation occurs, leading to a solid-liquid phase equilibrium, whereas, during gelation, only a semisolid phase exists because of a microscopic phase separation. Both crystallization and gelation are driven by supersaturation. Methods that result in supersaturation or add external energy to induce the formation of new phases can result in gelation or crystallization [9-14], such as cooling a hot solution, $\mathrm{pH}$ switch, ultrasonic treatment, and optical radiation. 
Dynamic arrest is regarded as the main mechanism of physical gelation [15], which means that a new phase induced by solute molecules is trapped in a kinetically favorable gel-like state rather than a thermodynamic stable state. The metastable state is the outcome of the competition between solute-solute interaction and solvent-solute interaction [16] and occurs in the metastable region in its phase diagram. For APIs, most studies focused on the metastable region of crystallization. Only a few reports involved the metastable equilibrium phase diagrams that contain the gel-like phase; meanwhile, gelation mechanism remains poorly understood.

In this paper, the gelation of a widely used erythromycin analog antibiotic, erythromycin ethylsuccinate (EES, CAS No. 1264-62-6, Fig. 1) is studied for the first time. First, the EES gel-like phase was characterized and the solvate nature of the fibers inside the phase was confirmed. Then, the EES gelation region was developed in the solvent system of 1-propanol. Finally, Fourier transform infrared (FTIR) spectra, nuclear magnetic resonance (NMR) spectra, and solvent parameters analyses were conducted to unravel the gelation mechanism at a molecular level. The results of this study provide useful guidelines to understand the gelation mechanism of other APIs.

\section{Experimental}

\section{Materials}

A white crystalline powder of EES $\left(\mathrm{C}_{43} \mathrm{H}_{75} \mathrm{NO}_{16}\right.$, MW 862.07 ) with a mass fraction purity of over $98 \%$ was purchased from Wuhan Yuancheng Gongchuang Science and Technology Ltd, China. The organic solvents (acetone, 1-propanol, etc., analytical reagent grade) were purchased from Tianjin Jiangtian Chemical Technology Co., Ltd, China, and were used without further purification. Deionized water was purchased from Tianjin QingYuanquan Purified

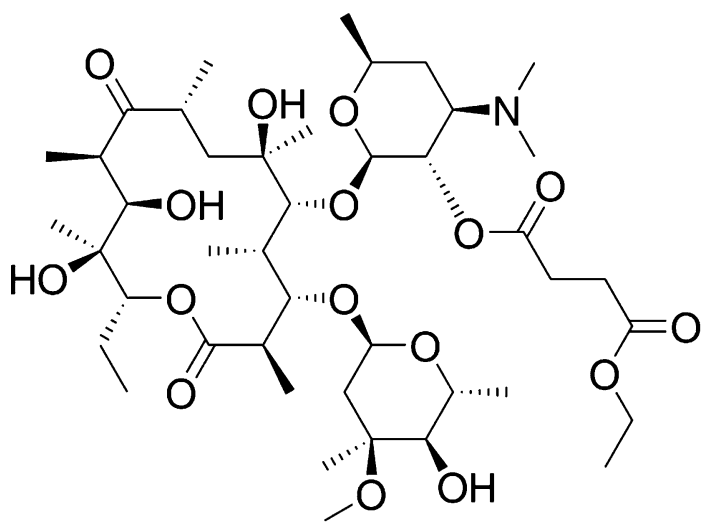

Fig. 1 EES molecular structure
Water Co., Ltd, China. Deuterated reagents (acetone-d6 and $\mathrm{D}_{2} \mathrm{O}, 99.9 \%$ deuterium content) were purchased from Tianjin Heowns Biochem Technology Co., Ltd, China.

\section{Preparation of Gel-Like Phase}

Method 1: Solventing-out. To achieve a gel-like phase, $1.5 \mathrm{~g}$ of EES powder was dissolved completely in $10 \mathrm{~mL}$ acetone and a transparent solution was formed at $20^{\circ} \mathrm{C}$. Under moderate mechanical stirring $(300 \mathrm{r} / \mathrm{min}), 6 \mathrm{~mL}$ water was added as an anti-solvent at a constant flowrate $(1.00 \mathrm{~mL} / \mathrm{min})$ using a peristaltic pump, and then the whole system turned into a gel-like state. Gelation also occurred in a 1-propanol-water mixture at different volume ratios.

Method 2: Cooling. 1.5 g EES powder was added into a $4 \mathrm{~g}$ water-acetone mixture $(1 \mathrm{~mol} / \mathrm{mol})$ and heated to $51^{\circ} \mathrm{C}$ to make the powder dissolve completely. Then, the hot solution was kept at room temperature to form a gel-like phase. Gelation also occurred in 1-propanol using a similar method.

Method 3: A gel-like phase was also formed when a moderate amount of EES powder was added to certain solvents. In a typical gelation process, $0.37 \mathrm{~g}$ EES powder was added into a $4 \mathrm{~g}$ water-acetone mixture $(1 \mathrm{~mol} / \mathrm{mol})$ at $20^{\circ} \mathrm{C}$ under $300 \mathrm{r} / \mathrm{min}$ stirring. After $5 \mathrm{~min}$, the solution became turbid and then a gel-like phase emerged. Gelation also occurred in 1-propanol when the amount of EES powder was changed.

\section{Solvent Screening}

The gelation of EES was investigated via method 1 in a series of solvents including methanol, ethanol, 2-propanol, 1, 2-propanediol, DMF, NMP, acetonitrile, formamide, cholamine, glycol, DMSO, triethanolamine, isopropyl ether, and THF. Water was used as an anti-solvent.

\section{Characterization}

Micrographs were acquired by scanning electron microscopy (Hitachi TM3000 tabletop SEM, Japan) and polarized optical microscopy (Olympus BX51, Japan). Powder X-ray Diffraction (PXRD) data were collected by a PXRD instrument (Rigaku D/MAX-2500, Japan) under the following conditions: $8^{\circ} /$ min scan speed, $0.02^{\circ}$ step size, $2^{\circ}-50^{\circ}$ scan range, and $\mathrm{Cu}$ $\mathrm{K} \alpha$ radiation $(\lambda=1.5406 \AA)$. Rheological experiments were conducted on a rheometer (Anton Paar MCR 302, Austria) at $20{ }^{\circ} \mathrm{C}$. Dynamic frequency sweep (DFS) was conducted in an angular frequency range of $100-0.1 \mathrm{rad} / \mathrm{s}$ with a strain amplitude of $0.05 \%$, and dynamic strain sweep (DSS) was conducted in a strain range of $0.01-100 \%$ at $10 \mathrm{rad} / \mathrm{s}$ frequency. Thermogravimetric analysis (TGA) and differential scanning calorimetry (DSC) were conducted on a TGA instrument (Mettler Toledo TGA/DSC 1/SF, Switzerland) and DSC instrument (Mettler Toledo DSC 1/500, Switzerland), 
respectively. 5-10 $\mathrm{mg}$ of samples was loaded in a crucible and heated from 25 to $250{ }^{\circ} \mathrm{C}$ at $10{ }^{\circ} \mathrm{C} / \mathrm{min}$ under $\mathrm{N}_{2}$ atmosphere. FTIR spectra were determined on a spectrometer (Bruker Tensor 27, Switzerland) with a resolution of $1 \mathrm{~cm}^{-1}$, and the solid powder was tableted with $\mathrm{KBr}$ and scanned in the range of $4000-400 \mathrm{~cm}^{-1}$. NMR spectra were determined on an NMR spectrometer (Bruker Avance III $400 \mathrm{MHz}$, Switzerland) in a $\mathrm{D}_{2} \mathrm{O}$ and acetone-d6 mixture $(1 \mathrm{~mol} / \mathrm{mol})$, which included ${ }^{13} \mathrm{C}$ NMR spectra and concentration variation ${ }^{1} \mathrm{H}-\mathrm{NMR}$ spectra, with a resolution of $0.45 \mathrm{~Hz}$ and sensitivity of 250:1.

\section{Measurement of the Phase Diagram}

\section{Solubility Measurement}

Dynamic Method $4 \mathrm{~g}$ of 1-propanol (designated as $m_{2}$ ) was poured into a 50-mL jacketed glass vessel, and a thermostat bath was used to regulate the experimental temperature. At a stirring rate of $300 \mathrm{r} / \mathrm{min}$, a certain small amount of EES powder was continuously added to the solvent to approach saturation. The last added amount was not more than $0.01 \mathrm{~g}$. The total amount of EES powder consumed when the slightly turbid solution did not turn transparent any more was recorded as $m_{1}$. Each measuring point was repeated thrice, and the solubility of EES was calculated by Eq. (1):

$x=\frac{m_{1}}{m_{2}}$,

The temperature ranged from 10 to $50^{\circ} \mathrm{C}$, and 5 equidistant points were considered.

Static Method The cloudiness of the slightly turbid solution mentioned above increased with time, suggesting a phase transition after the EES dissolution. Thus, the static method was used to acquire the solubility data and the equilibrium solid phase was tested. The experiments were carried out in Erlenmeyer flasks, which were shaken in a thermostat shaking bath at $300 \mathrm{r} / \mathrm{min}$. Excessive EES powder and $15 \mathrm{~g} 1$-propanol were added into the Erlenmeyer flasks, and samples were taken every $2 \mathrm{~h}$. After $8 \mathrm{~h}$, a liquid-solid equilibrium was reached, and then the shaking was stopped for $0.5 \mathrm{~h}$ for the suspension to separate into two phases. A total sample of $5 \mathrm{~mL}$ was extracted with a syringe equipped with a $0.22-\mu \mathrm{m}$ polyvinylidene fluoride filter. The sample was placed in an evaporating dish, and the weight of the dish $\left(X_{0}\right)$ and the total weight of the dish and solution $\left(X_{2}\right)$ were recorded. The liquid samples were evaporated in a vacuum oven at $50{ }^{\circ} \mathrm{C}$ and weighed every $12 \mathrm{~h}$ until a constant weight $\left(X_{1}\right)$ was achieved. The PXRD pattern of the wet solid phase was recorded. The solubility of the equilibrium solid phase was calculated by Eq. (2):

$x=\frac{X_{1}-X_{0}}{X_{2}-X_{1}}$,
The samples were measured thrice for each Erlenmeyer flask, and the average value was used to calculate the solute solubility. The temperature ranged from 10 to $50{ }^{\circ} \mathrm{C}$ over 5 equidistant points.

\section{Gelation Points Measurement}

Cooling Method An appropriate amount of EES powder $\left(m_{1}\right)$ and $6 \mathrm{~g}$ of 1-propanol $\left(m_{2}\right)$ were added into a $50-\mathrm{mL}$ jacketed glass vessel within a thermostat bath. Under a $300 \mathrm{r} / \mathrm{min}$ stirring rate, the vessel was heated at $1{ }^{\circ} \mathrm{C} / \mathrm{min}$ to dissolve EES completely, and then, it was cooled at the same rate. The temperature was recorded when the solution became discernibly turbid. This was assigned as the gelation point of EES, after which the sample would completely turn into a gel-like phase. The amount of EES powder was then changed, and the experiments were repeated to obtain a series of gelation points at different concentrations. The concentration of the solution was calculated by Eq. (1).

\section{Results and Discussion}

\section{Gelation Phenomenon and Characterization of the EES Gel-like Phase}

The EES gel-like phase obtained through three methods showed a white opaque appearance and exhibited no gravitational flow under tube inversion test. When heated, the gel-like phase was separated into solid and liquid phases and eventually turned into a transparent solution. When the solution was cooled, the gel-like state reappeared, as shown in Fig. 2. The transition temperatures depended on the solution concentration and solvent composition.

Long, thin, and flexible fibers were observed in the microscope images of the gel-like phase, and their sizes varied with the preparation methods (Fig. S1-S3). The variation of the microscale morphology (Fig. 3) of the gel-like phase was tracked throughout the gelation process by a microscope. On the initial stage, the emergence of a few single fibers caused

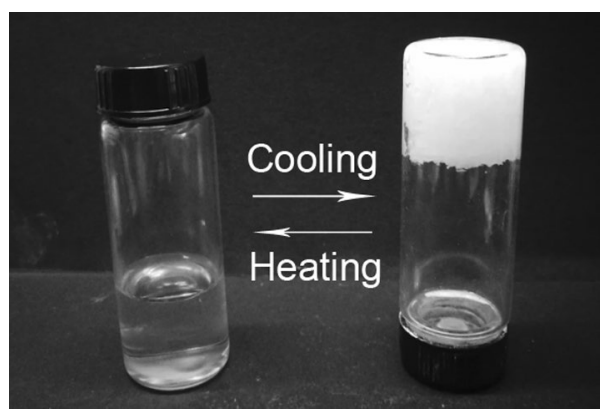

Fig. 2 Reversible thermal transition of EES gel-like phase 

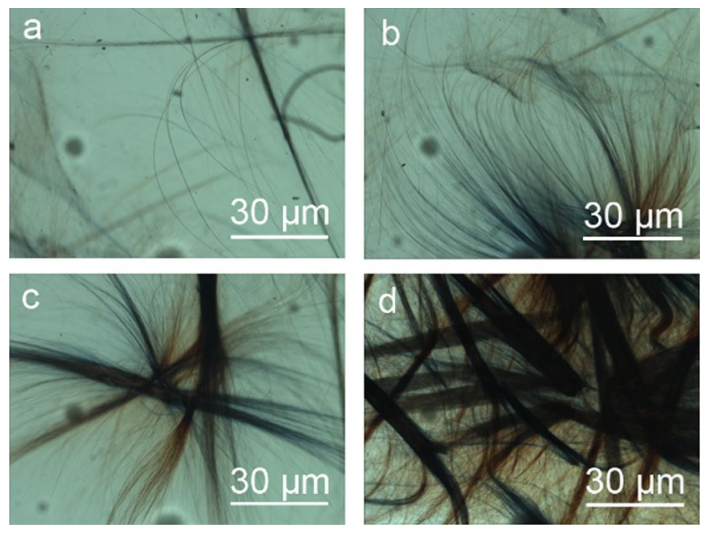

Fig. 3 Microscope images of the EES gel-like phase. a Single fibers, b fiber clusters, c twisted and cross-linked clusters, and d 3D fibrillar network

a slight turbidity of the solution. By keeping the temperature and solvent content constant, more fibers emerged and gathered to form fiber clusters. The clusters twisted and crosslinked with each other to generate a 3D network structure.

The mechanical properties of the gel-like phase were measured by dynamic rheology characterization. The DSS curves of the EES gel-like phase are shown in Fig. 4a. Both storage modulus $\left(G^{\prime}\right)$ and loss modulus $\left(G^{\prime \prime}\right)$ remained
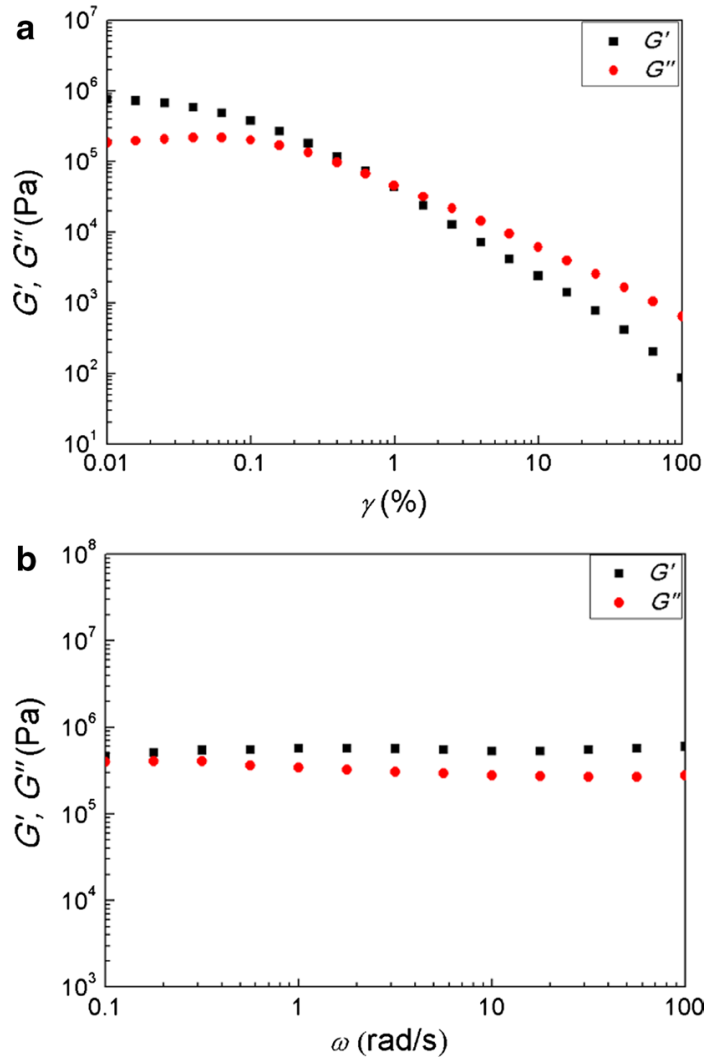

Fig. 4 Rheological curves of the gel-like phase. a DSS and b DFS constant with the increase in strain from 0.01 to $0.1 \%$, which is the linear viscoelastic region (LVR) of the gel-like phase. The low strain limit $(0.1 \%)$ demonstrated the crystalline nature of the fibers [17]. The DFS was performed within the LVR, as shown in Fig. 4b. In the whole frequency range, $G^{\prime}$ was larger than $G^{\prime \prime}$, and both were almost independent of frequency, meaning that the sample was unable to flow and remained in a solid-like phase regardless of the bearing force. These rheological properties are similar to those of typical gel substances [18].

\section{Solvatomorphs and Gelation Mechanism of EES}

\section{Analysis of PXRD Patterns}

The morphology of the fibers was maintained when the EES gel-like phase was dried in air for $1 \mathrm{~h}$, while the immobilized solution was completely dried out. The PXRD patterns of EES fibers are shown in Fig. 5, where the series of diffraction peaks in the PXRD patterns indicate the crystalline nature of the fibers. The fibers formed in different solvents showed the same PXRD patterns, indicating identical packing modes of the EES molecules in the crystal structure. The obtained Bragg lattice spacing values $(d)$ of $1.99,0.97$, $0.63,0.55 \mathrm{~nm}$ closely followed the ratio of $1: 1 / 2: 1 / 3: 1 / 4$, suggesting the existence of an 1D lamellar structure in the nanofibers of the gel-like phase with a layer distance of $1.99 \mathrm{~nm}[19,20]$.

Compared with the PXRD pattern of EES crystals, the PXRD patterns of the fibers showed two new peaks at $4.44^{\circ}$ and $13.96^{\circ}$, and the peaks at $8.15^{\circ}, 11.05^{\circ}, 15.22^{\circ}, 17.00^{\circ}$, and $20.68^{\circ}$ disappeared, suggesting that the crystalline form of the fibers is different from that of the EES crystals and has not been reported. After drying the samples in a vacuum oven at $50{ }^{\circ} \mathrm{C}$ for $24 \mathrm{~h}$, the PXRD patterns of the dried samples (Fig. S4) were different from those of the fibers but

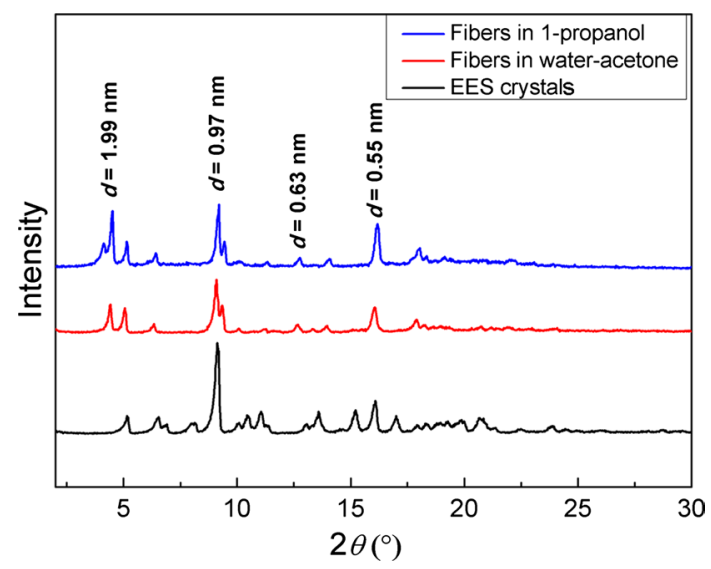

Fig. 5 PXRD patterns of EES crystals (black); EES fibers in wateracetone (red) and 1-propanol (blue) 
similar to that of the EES crystals. The SEM image of a dried sample (Fig. S5) shows a similar morphology to that of the EES crystals. The changes indicate that the fibers transformed to EES crystals.

\section{Thermal Analysis}

Figure 6 shows the thermal analysis of EES fibers. The TGA curve of the fibers formed in 1-propanol showed a weight loss of $2.55 \mathrm{wt} \%$, and the DSC curve had an endothermic peak at the corresponding temperature range, indicating that the sample was 1-propanol solvate. For the fibers formed in the water-acetone mixture, a weight loss of $0.90 \mathrm{wt} \%$ and an endothermic peak appeared in TGA and DSC curves in the same temperature range, respectively, proving that the sample was also a kind of EES solvate.

The headspace sampling gas chromatography (GC) analysis verified the existence of acetone and 1-propanol in the fibers formed in water-acetone and 1-propanol, respectively (Figs. S6 and S7). The existence of water in the fibers formed in the water-acetone mixture was proved by volumetric Karl Fischer titration (see Supporting Information). The results
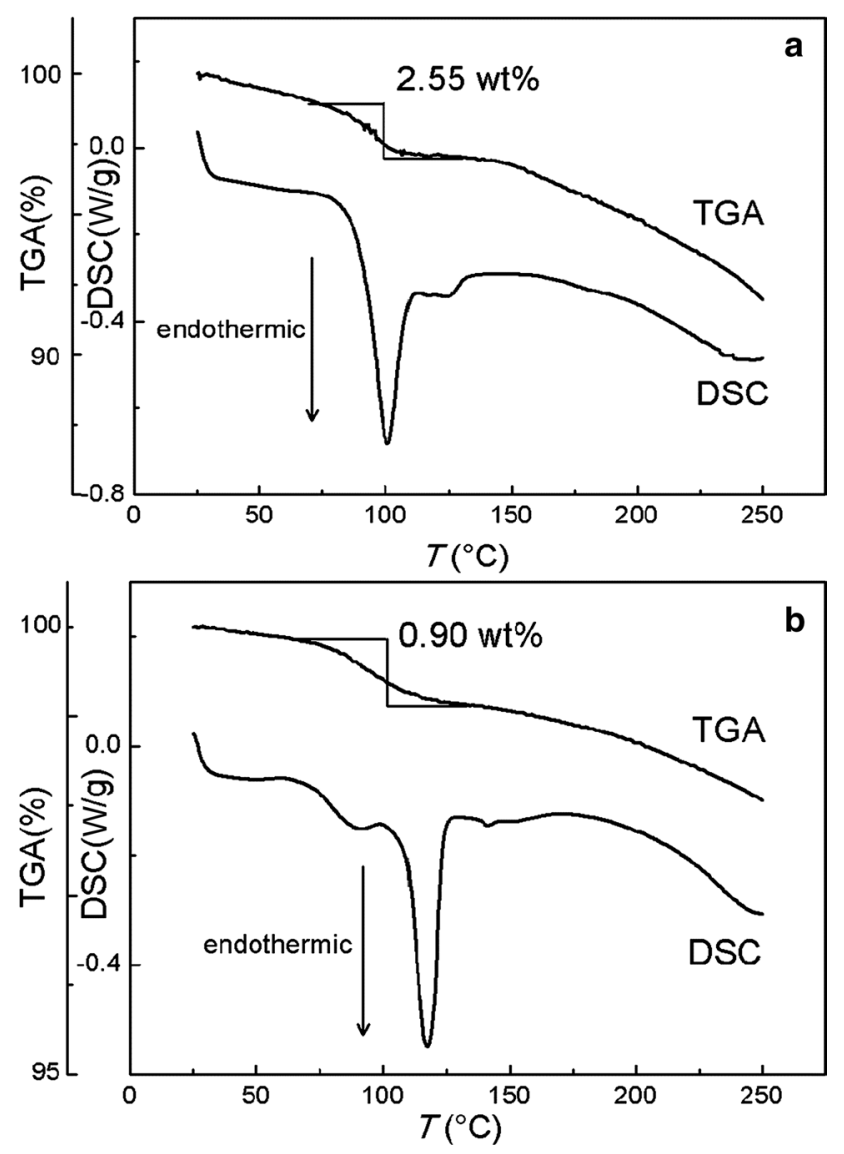

Fig. 6 DSC and TGA curves of EES fibers. a fibers in 1-propanol and b fibers in water-acetone obtained from the above two sections indicate that the fibers formed in different solvents were EES solvatomorphs, which were discovered for the first time. The gelation of the EES in its solution was due to the formation and cross-linking of the fibrous solvates.

\section{Conditions for Gelation}

\section{Phase Diagram}

The solubility data in 1-propanol were measured by both static and dynamic methods, corresponding to the blue and red curves in Fig. 7. The equilibrium solid phase in the static method was proved to be EES 1-propanol solvate (Fig. S8), demonstrating the solubility data (blue curve) corresponding to the solubility of EES 1-propanol solvate. At the same temperature, the solubility of the EES solvate is lower than that of the EES crystals. Thus, with the addition of the EES crystals into 1-propanol, the EES crystals transformed into a solvate when the concentration of the EES in solution exceeded the solubility of the solvate. In a series of typical experiments, solutions represented by points from $L_{1}$ to $J_{1}$ were prepared. It showed that because few fibrous crystalline solvates crystallized out under low supersaturation, the solution could not gel in the region between the blue and red curves, which can be regarded as the crystallization region. Gelation would inevitably occur when the concentration is above the red curve, which can be regarded as the gelation region. The lower the supersaturation in the gelation region, the more difficult and time-consuming it is for gelation to occur. Thus, it is indicated that a high degree of supersaturation promotes gelation. The gelation method 3 described in Experimental section is hence termed as "trans-crystal gelation," and the solubility of the EES crystals can be regarded as the critical gelation concentration. The black curve in Fig. 7 corresponds to the gelation points of EES measured

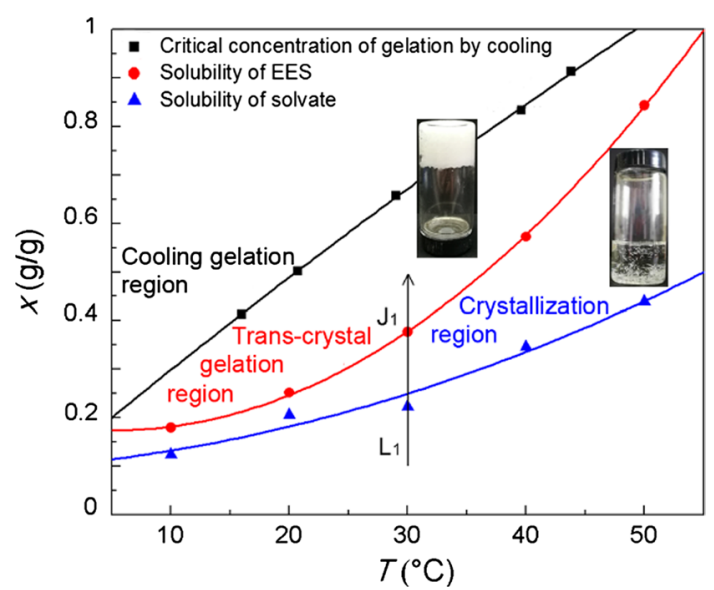

Fig. 7 Phase diagram of EES in 1-propanol 
by cooling. Compared with the trans-crystal gelation region, the solution gels fast in the cooling gelation region, and the critical concentration of cooling gelation is higher than that of trans-crystal gelation.

\section{Analysis of Solvent Parameters}

The influence of solvent properties on the EES gelation was investigated by solvent screening. The solutions of EES in acetone, methanol, ethanol, 1-propanol, 2-propanol, DMF, and NMP gelled entirely with added water, while the solution in acetonitrile gelled only partially. As shown in Table 1, the solvents that facilitated gelation have something in common: a dielectric constant range of $18.3-37.5, \delta_{\mathrm{p}}$ (dipole interactions) of $6.1-18 \mathrm{MPa}^{1 / 2}$ in Hansen solubility parameters, and $\pi^{*}$ (polarizability parameter) of $0.48-0.88$ in Kamlet-Taft solubility parameters [21]. Outside these ranges, it is impossible for gelation to occur in the corresponding solvents.

The matching degree of solvent-solute intermiscibility can be described by the residual of solubility $\Delta \delta$. A smaller value of $\Delta \delta$ means a better solubility of a solute in a solvent. $\Delta \delta$ can be calculated from Eq. (3):

$\Delta \delta=\sqrt{\left(\delta_{\mathrm{d}_{1}}-\delta_{\mathrm{d}_{2}}\right)^{2}+\left(\delta_{\mathrm{p}_{1}}-\delta_{\mathrm{p}_{2}}\right)^{2}+\left(\delta_{\mathrm{h}_{1}}-\delta_{\mathrm{h}_{2}}\right)^{2}}$,

where $\delta_{\mathrm{d}}$ and $\delta_{\mathrm{h}}$ represent the dispersion interactions and hydrogen bond interactions, respectively, while subscripts 1 and 2 correspond to EES and solvent, respectively. Table 1 shows the $\Delta \delta$ of the studied gelation solvents, and it ranges from 4.3 to $14 \mathrm{MPa}^{1 / 2}$, which is smaller than those of other solvents, indicating that the gelation solvents have a better dissolving capacity for EES. Combined with the analysis of solubility parameters, it can be concluded that a high solubility of EES in the solvents is necessary for gelation. A low solubility results in the separation of a small amount of fibers from the solution, which makes it hard to trap quantities of solvent molecules and ultimately leads to crystallization.

\section{Intermolecular Interaction Analysis}

\section{FTIR}

FTIR characterization was performed on the EES crystals and fibers. The peaks at 1741 and $1698 \mathrm{~cm}^{-1}$ in Curve a of Fig. 8 represent the $\mathrm{C}=\mathrm{O}$ stretching vibration of ester groups and carbonyl of EES, respectively. For fibers, the former peak shifted to $1736 \mathrm{~cm}^{-1}$, while the latter disappeared, which could have been masked by the former. It was speculated that ester groups participated in the intermolecular hydrogen bonds as $\mathrm{H}$ acceptors, accepting $\mathrm{H}$ from the solvent molecules to form the EES solvate fibers. Furthermore, the red shift of $\mathrm{C}-\mathrm{O}-\mathrm{C}$ vibration peaks in the ester groups and glucosidic bonds also imply the participation of these $\mathrm{O}$ atoms in the supramolecular self-assembly of EES solvate fibers as $\mathrm{H}$ acceptors (Table $\mathrm{S} 1$ ).
Table 1 Gelation behaviors of EES in different solvents and solvent parameters analysis

\begin{tabular}{|c|c|c|c|c|c|c|c|c|}
\hline \multirow[t]{2}{*}{ No. } & \multirow[t]{2}{*}{ Solvent } & \multirow[t]{2}{*}{$\begin{array}{l}\text { Gelation } \\
\text { behavior }\end{array}$} & \multirow[t]{2}{*}{$\begin{array}{l}\text { Dielectric con- } \\
\text { stant }[21]\left(25^{\circ} \mathrm{C}\right)\end{array}$} & \multicolumn{3}{|c|}{$\begin{array}{l}\text { Hansen solubility } \\
\text { parameters }\left(\mathrm{MPa}^{1 / 2}\right) \\
{[21,22]}\end{array}$} & \multirow[t]{2}{*}{$\begin{array}{l}\Delta \delta \\
\left(\mathrm{MPa}^{1 / 2}\right)\end{array}$} & \multirow[t]{2}{*}{$\pi^{*}[21]$} \\
\hline & & & & $\delta_{\mathrm{d}}$ & $\delta_{\mathrm{p}}$ & $\delta_{\mathrm{h}}$ & & \\
\hline 1 & Acetone & G & 20.7 & 15.5 & 10.4 & 7 & 4.306 & 0.62 \\
\hline 2 & Methanol & G & 31.2 & 15.1 & 12.3 & 22.3 & 14.08 & 0.6 \\
\hline 3 & Ethanol & G & 25.7 & 15.8 & 8.8 & 19.4 & 10.61 & 0.54 \\
\hline 4 & Propanol & G & 22.2 & 16 & 6.8 & 17.4 & 8.693 & 0.52 \\
\hline 5 & 2-Propanol & G & 18.3 & 15.8 & 6.1 & 16.4 & 7.782 & 0.48 \\
\hline 6 & DMF & G & 36.71 & 17.4 & 13.7 & 11.3 & 7.778 & 0.88 \\
\hline 7 & NMP & G & 32 & 18 & 12.3 & 7.2 & 7.077 & \\
\hline 8 & Acetonitrile & PG & 37.5 & 15.3 & 18 & 6.1 & 11.15 & 0.66 \\
\hline 9 & Formamide & $\mathrm{P}$ & 111 & 17.2 & 26.2 & 19 & 21.49 & 0.97 \\
\hline 10 & Cholamine & $\mathrm{P}$ & 37.72 & 17.2 & 15.6 & 21.3 & 15.1 & \\
\hline 11 & Glycol & $\mathrm{P}$ & 37.7 & & & & & 0.92 \\
\hline 12 & DMSO & $\mathrm{P}$ & 48.9 & 18.4 & 16.4 & 10.2 & 10.36 & 1 \\
\hline 13 & Triethanolamine & $\mathrm{P}$ & 29.36 & & & & & \\
\hline 14 & Isopropyl ether & $\mathrm{P}$ & 4.49 & & & & & \\
\hline 15 & Water & $\mathrm{P}$ & 7.58 & 16.8 & 5.7 & 8 & 4.2 & 0.55 \\
\hline 16 & THF & $\mathrm{P}$ & 78.5 & 15.6 & 16 & 42.3 & 34.26 & 1.09 \\
\hline 17 & EES & & & 13.2 & 7.5 & 9.2 & - & \\
\hline
\end{tabular}

$\mathrm{G}, \mathrm{PG}$, and $\mathrm{P}$ represent gelation, partial gelation, and precipitate, respectively 


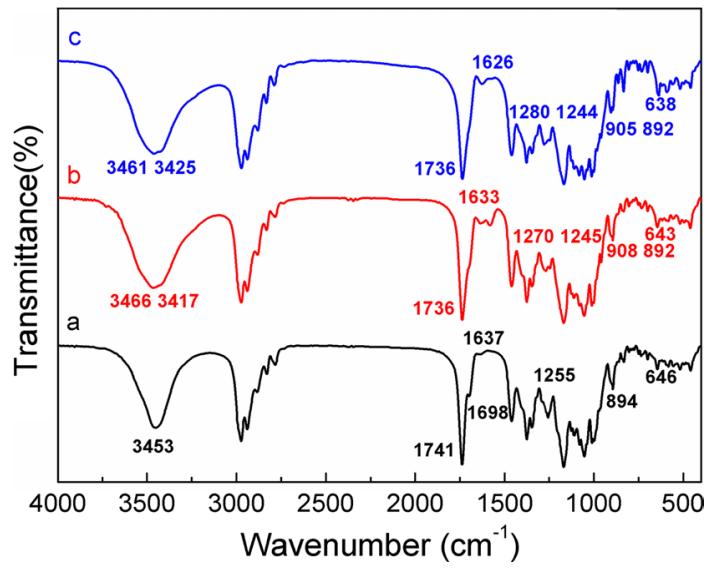

Fig. 8 Solid FTIR absorption curves. a EES crystals, b fibers in water-acetone, and $\mathbf{c}$ fibers in 1-propanol

The wide peak of $-\mathrm{OH}$ stretching vibration at $3453 \mathrm{~cm}^{-1}$ of EES crystals indicates that the EES molecules interacted with each other through hydrogen bonds between hydroxyls. For fibers, the $-\mathrm{OH}$ stretching vibration peak split into two peaks, indicating the variation of intermolecular hydrogen bonds between the hydroxyl groups of the EES molecules after fibers formation. Some of the EES-EES intermolecular hydrogen bonds between the hydroxyls were substituted by the new EES-solvent intermolecular hydrogen bonds in the supramolecular fibers [23, 24], as the small size makes the solvent molecules bond easily with the EES molecules. Moreover, both the out-of-plane bending vibration peak of $-\mathrm{OH}$ and the bending vibration peak of $-\mathrm{OH}$ on the cladinose ring exhibited a red shift in the fibers, demonstrating the participation of these hydroxyls in intermolecular hydrogen bonds.

Because of the presence of many intermolecular hydrogen bonds between the EES and solvent molecules, they assembled together to form 1D lamellar structural units, which assembled into 2D fibers quickly, and many branches were generated around the fibers, as shown in Fig. 9. Finally, the fibers intertwined and cross-linked with each other to construct a 3D self-assembled fibrillar

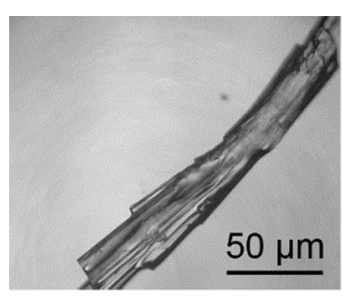

a

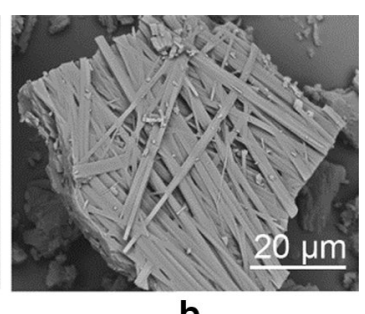

b
Fig. 9 Images of fibrous crystals in 1-propanol. a optical microscope of crystal and $\mathbf{b}$ SEM image of fibers network structure. Thus, the formation and growth of the EES-solvent supramolecular fibers is in accordance with the mechanism of crystallographic mismatch branching [25].

\section{NMR}

Concentration variation ${ }^{1} \mathrm{H}$ NMR spectra are shown in Fig. 10. With the increase in EES concentration, the system changed from a solution to gel-like state, which led to the weakening of peak intensity and peak splitting, suggesting that the mobility of molecules decreased, and protons could not produce individual sharp signals as they were in the aggregated state in the gel-like phase [26]. The $\mathrm{H}$ atoms of $\mathrm{H}_{2} \mathrm{O}$ ( $\delta$ of $\sim 4.30 \mathrm{ppm}$ ) show downfield chemical shifts with the increasing EES concentration, indicating the participation of $\mathrm{H}_{2} \mathrm{O}$ molecules in the EES-solvent hydrogen bonds as $\mathrm{H}$ donors.

The ${ }^{13} \mathrm{C}$ NMR spectra (see Fig. S10) were also recorded for the EES solution $(1.875 \mathrm{mg} / \mathrm{mL})$ and gel-like state $(3.75 \mathrm{mg} / \mathrm{mL})$. The chemical shift at nearly $\delta=211.5 \mathrm{ppm}$ (Fig. 11a) corresponds to the $\mathrm{C}$ atoms of carbonyl groups. As the amount of solvents was much larger, we assigned the high peak to acetone-d6 and the low peak to the carbonyl group on the EES ring. The downfield shifts of these peaks indicate the participation of carbonyl groups as $\mathrm{H}$ acceptors in the EES-solvent intermolecular hydrogen bonds. In addition, the peaks of -COOC- in ester groups around $175 \mathrm{ppm}$ (Fig. 11b) showed minute downfield shifts (0.06-0.1 ppm), suggesting the involvement of ester groups in the EES-solvent intermolecular hydrogen bonds.

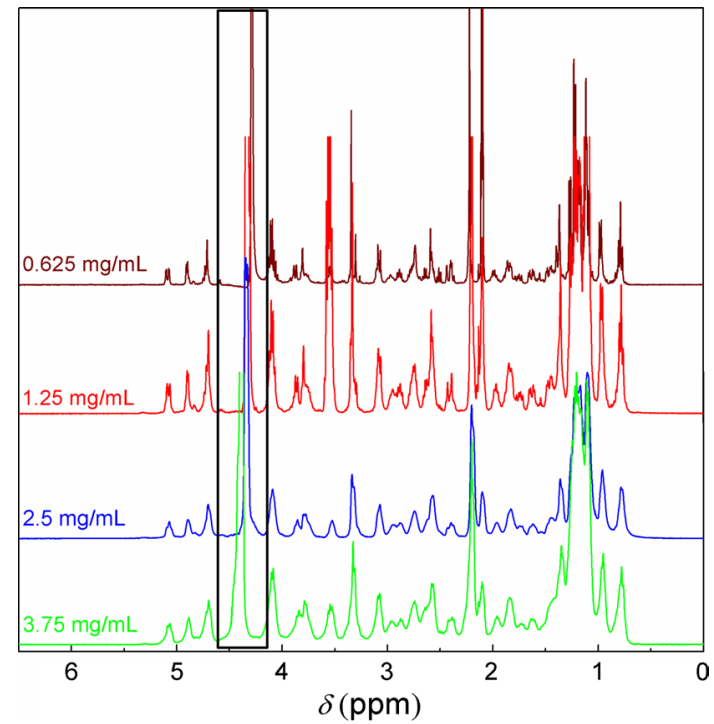

Fig. 10 Concentration variation ${ }^{1} \mathrm{H}$ NMR spectra of EES in $\mathrm{D}_{2} \mathrm{O}$ :acetone-d6 $(v: v=3: 5)$ 


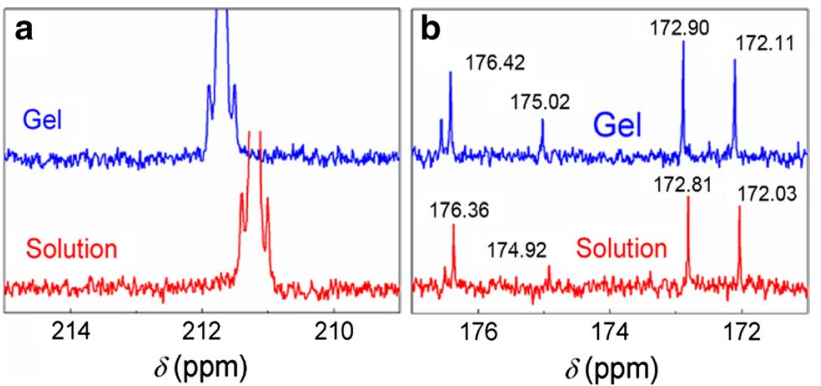

Fig. 11 Partial ${ }^{13} \mathrm{C}$ NMR spectra of EES in $\mathrm{D}_{2} \mathrm{O} /$ acetone-d6 $(v: v=3: 5)$

\section{Conclusions}

Gelation phenomenon of EES in single or mixed solvents was reported for the first time in this paper. Analyses of PXRD, TGA/DSC, and GC indicate that the fibers formed during the gelation process were solvates of EES, which were discovered for the first time. The phase diagram of the gelation region and crystallization region was obtained for EES in 1-propanol. High supersaturation was found to be the reason for the formation of numerous fibrous solvate and gelation. Solvent parameters analysis indicated that solvent polarity was also a key factor for gelation. The results showed that gelation can be avoided by controlling the supersaturation and selecting appropriate solvents. FTIR and NMR analyses suggested that the dominant driving force for the formation of EES solvate fibers was the intermolecular hydrogen bonds between EES and solvent molecules. The crystalline fibers intertwined with each other to construct a 3D fibrillar network, immobilizing solvent molecules and leading to the formation of a gel-like phase.

Acknowledgements This study was supported by the National Natural Science Foundation of China (Nos. 21576187, 21776203).

Open Access This article is distributed under the terms of the Creative Commons Attribution 4.0 International License (http://creativeco mmons.org/licenses/by/4.0/), which permits unrestricted use, distribution, and reproduction in any medium, provided you give appropriate credit to the original author(s) and the source, provide a link to the Creative Commons license, and indicate if changes were made.

\section{References}

1. Saboktakin MR, Tabatabaei RM (2015) Supramolecular hydrogels as drug delivery systems. Int J Biol Macromol 75:426-436

2. Zhao F, Ma ML, Xu B (2009) Molecular hydrogels of therapeutic agents. Chem Soc Rev 38:883-891

3. Song LC (2011) Study on purification crystallization technology of clopidogrel hydrogen sulfate (Form I). Dissertation, Tianjin University, China (in Chinese)

4. Liu Y (2010) The study on refining process of valsartan. Dissertation, Tianjin University, China (in Chinese)
5. Estroff LA, Hamilton AD (2004) Water gelation by small organic molecules. Chem Rev 104:1201-1218

6. Dastidar P (2008) Supramolecular gelling agents: can they be designed? Chem Soc Rev 37:2699-2715

7. Song J, Sun HJ, Sun SJ et al (2013) Synthesis and gel properties of sorbitol derivative gelators. Trans Tianjin Univ 19:319-325

8. Zhang Y, Weiss RG (2017) How do H-bonding interactions control viscoelasticity and thixotropy of molecular gels? Insights from mono-, di- and tri-hydroxymethylated alkanamide gelators. J Colloid Interface Sci 486:359-371

9. Gong JB, Wang JK, Wei HY (2005) Effect of mixed solvents and additives on the habit modification of 6-APA crystals. Trans Tianjin Univ 11:157-161

10. Chen Y, Gao YY, Zhu L et al (2010) Metastable state of clindamycin phosphate in cooling crystallization. Trans Tianjin Univ $16: 142-146$

11. Liu J, Xu FY, Sun ZL et al (2016) A supramolecular gel based on a glycosylated amino acid derivative with the properties of gel to crystal transition. Soft Matter 12:141-148

12. Martin AD, Wojciechowski JP, Bhadbhade MM et al (2016) A capped dipeptide which simultaneously exhibits gelation and crystallization behavior. Langmuir 32:2245-2250

13. Singh VK, Behera B, Pramanik K et al (2015) Ultrasonicationassisted preparation and characterization of emulsions and emulsion gels for topical drug delivery. J Pharm Sci 104:1035-1044

14. Ran X, Wang HT, Zhang P et al (2011) Photo-induced fiber-vesicle morphological change in an organogel based on an azophenyl hydrazide derivative. Soft Matter 7:8561-8566

15. Anderson VJ, Lekkerkerker HNW (2002) Insights into phase transition kinetics from colloid science. Nature 416:811-815

16. Vintiloiu A, Leroux JC (2008) Organogels and their use in drug delivery - a review. J Controlled Release 125:179-192

17. Terech P, Pasquier D, Bordas V et al (2000) Rheological properties and structural correlations in molecular organogels. Langmuir $16: 4485-4494$

18. Wang YJ, Tang LM, Yu J (2008) Investigation of spontaneous transition from low-molecular-weight hydrogel into macroscopic crystals. Cryst Growth Des 8:884-889

19. Mallia VA, Terech P, Weiss RG (2011) Correlations of properties and structures at different length scales of hydro- and organo-gels based on $\mathrm{N}$-alkyl-(R)-12-hydroxyoctadecylammonium chlorides. J Phys Chem B 115:12401-12414

20. Yu Y, Wang S, Jia L et al (2016) Organogels from different selfassembling novel L-proline dihydrazide derivatives: gelation mechanism and morphology investigations. J Sol-Gel Sci Technol 78:218-227

21. Reichardt C, Welton T (2011) Solvents and solvent effects in organic chemistry. Wiley-VCH Verlag, Weinheim, Germany

22. Li X (2016) A study on the thermodynamic and kinetic characteristics of oiling-out crystallization process of erythromycin ethylsuccinate. Dissertation, Tianjin University, China (in Chinese)

23. Iwasaki H, Sugawara Y, Adachi T et al (1993) Structure of 6- $O$-methylerythromycin A (clarithromycin). Acta Crystallogr Sect C 49:1227-1230

24. Brüning J, Trepte TK, Bats JW et al (2012) Erythromycin A dimethyl sulfoxide disolvate 1.43-hydrate. Acta Crystallogr Sect E 68:700-701

25. Liu XY (2005) Gelation with small molecules: from formation mechanism to nanostructure architecture. In: Fages F (ed) Low molecular mass gelators. Springer, Berlin, Germany, pp 1-37

26. Zhang M, Waldron KC, Zhu XX (2016) Formation of molecular hydrogels from a bile acid derivative and selected carboxylic acids. RSC Adv 6:35436-35440 